\title{
An Enhanced AODV Protocol for External Communication in Self-Driving Vehicles
}

\author{
Khattab M. Ali Alheeti \\ School of Computer Sciences and Electronic Engineering \\ University of Essex, Colchester, UK \\ University of Anbar, College of Computers-Anbar, Iraq \\ kmali@.essex.ac.uk
}

\begin{abstract}
The increasing number of autonomous and semiautonomous vehicles on the road leads to an increasing need for external vehicle communication, in particular through emerging vehicular ad hoc networks also known as VANETs. This technology has the ability to facilitate intelligent transportation applications, comfort and other required services for self-driving vehicles. However, suitable routing protocols need to be utilised in order to provide stable routing and enable high performance for this external communication in autonomous vehicles. Ad hoc on Demand Distance Vector routing (AODV) is to date rarely used in mobile ad hoc network but offers great potential as a reactive routing protocol. However, the AODV protocol is affected by poor performance, when directly employed in VANETs. In this paper, two improvements are presented to the route selection and route discovery of AODV to improve its performance in forms of packet delivery rate and communication link stability for VANETs. Thus, we obtain new vehicle V-AODV that suits the specific requirements of autonomous vehicles communications. Simulation results demonstrate that V-AODV can enhance the route stability, reduce overhead and improve communication performance between vehicles.
\end{abstract}

Keywords-routing protocol, Ad hoc on Demand Distance Vector protocol, vehicular ad hoc network, autonomous vehicles.

\section{INTRODUCTION}

Autonomous and semi-autonomous vehicles are increasingly perceived as means to improve passenger safety and protection from driver's errors. The function of these vehicles is supported by the communication system transmitting control data, sensitive information and communication data between vehicles and Road Side Unit (RSUs). The communication systems of autonomous vehicles can be classified into internal and external communication systems. Vehicular ad hoc networks (VANETs) are typically employed as external communication system of driverless vehicles [1]. These emerging new networks integrate the capabilities of new wireless networks types to autonomous vehicles. The ultimate goal of this VANET technology is to offer ubiquitous connectivity for drivers/passengers travelling on the road. In addition, these networks provide a variety of applications for comfort, efficiency and safety of traffic and driver assistance which can be shared among vehicles in the radio coverage area.

VANETs networks can be divided into two types [2]:

- Vehicle-to-Vehicle Communications (V2V): Autonomous vehicles have the ability to establish communication wirelessly with another mobile node in that radio communication zone.

- Vehicle-to-RSUs (V2R): This communication between the autonomous vehicles and their fixed infrastructure/ RSUs

\author{
Klaus McDonald-Maier \\ School of Computer Sciences and Electronic Engineering \\ University of Essex, Colchester, UK \\ kdm@essex.ac.uk
}

on the road side. This is utilised in order to track management services and traffic.

An overview of the external communication for self-driving vehicles is shown in figure 1.

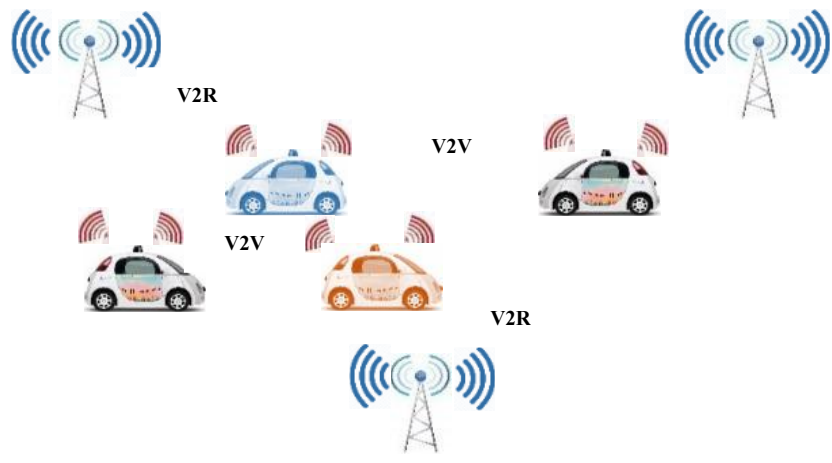

Fig. 1: Communication system of autonomous vehicles [3].

Vehicles with wireless equipment in VANETs form what is known as a dynamic network, which gives them the ability to communicate with others during their movement along the road. This direct communication between vehicles makes it possible for vehicles to exchange messages with one another even in a situation where there are no fixed infrastructures like access points of wireless dedicated access networks or RSUs [4]. The function of these radio-enabled vehicles is to act in similar way like mobile nodes as Mobile as hoc Networks (MANETs) [5].

VANETs are effectively a special type of MANET and they this share some characteristics with MANETs [6]. One similarity is that there are no fixed infrastructures in the networks, hence nodes in the network can exchange message with one another without the support of the infrastructure. Additionally, the network's topology is changing continuously, because all the nodes in both types of networks are moving.

Furthermore, the range of communication between nodes is limited which implies that mobile nodes may need to send messages to their destination using multiple hops with the aid of other intermediate nodes, instead of exchanging messages with destination directly [6]. However, VANETs have some special characteristics that are different from MANETs. One difference is that the nodes in MANETs mostly move randomly without spatial constraints [7]. On the other hand, VANETs of mobile vehicle can only travel along fixed paths as the streets and roads which act as constraints. Therefore, the topology of VANETs can be predicted to some mobile degree. Another major difference is 
that the topology of VANETs has the ability to change much faster than a typical MANET; this is possible due to the high speed of mobile vehicular nodes. A third factor is that vehicles are typically equipped with on-board sensors, for instance GPS receiver.

These on-board sensors can be utilised to provide speed information, direct the vehicle and even provide location information for the vehicle. However, performing routing in VANETs is a complex task due to these characteristics. There are several popular routing protocols in MANETs [8] [9] [9]. However, the direct application of existing routing protocols to VANETs may result in poor performance due to the differences between the two networks [10]. Ad hoc on Demand Distance Vector routing (AODV) which is one of the most popular routing protocols in MANETS, requires improvements to be utilised effectively in VANETs [11] [12].

In this paper, the AODV routing protocol is improved to be more compatible with the external communication of self-driving vehicles. Specifically, the route discovery phase and route selection phase of AODV protocol are optimised for use in VANETs. In addition, each mobile node is equipped with direction information, speed and position information for each vehicle.

In our paper, the proposed VANET-AODV requires two steps:

1. Forward Route Request (RREQ) packets are selected for the vehicles. These mobile nodes have been selected because of their stable links with others. The control overhead is reduced at the same time because not every neighbour vehicle is utilised to forward RREQ messages.

2. The most stable route for packet transition is utilised when the source vehicle receives multiple routes to destination vehicle.

The remainder of this paper is organised as follows: related works are discussed in section two; an overview of AODV is provided in section three; section four discusses the proposed VANET-AODV routing protocol; the methodology of the proposed routing protocol is presented in section five, and results of the simulation are presented in section six. The final section of our paper offers conclusion and future work.

\section{RELATED WORKS}

The emergence of autonomous vehicles which used VANETs has attracted significant interest due to its potentially positive impact on society. Routing protocols are considered a vital for these vehicles which were depended on these to move and exchange warning messages, Cooperative Awareness Messages (CAM), notification messages, control data and sensitive information. Routing protocols of self-driving and semiautonomous vehicles have been widely researched.

Ben et al. [13] suggested an enhanced AODV routing protocol for use in vehicles. The proposed routing protocol has the ability to adapt with the high dynamic topology of vehicles. The authors enhance the route stability and reduce the control head of VANETs. In addition, their proposed AODV is characterized by high Packet Delivery Rate (PDR) and fewer broken communication links.

The authors of [14] proposed a routing protocol Improved AODV (IAODV) to VANETs. It can provide accurate information from vehicles to other vehicles in that radio zone. IAODV has limited source routing to hop between source vehicle to destination vehicle. The performance of the proposed IAODV is compared with traditional AODV under certain conditions to measure packet delivery rate, end-to end delay and normalisation routing load.

Abbs et al. in [15] proposed to enhance the AODV reaction routing protocol's performance. The enhancing process is based on fuzzy system that select most trusted node to establish a path between source to destination. The proposed AODV has the ability to reduce the battery cost of the routing protocol and improve PDR, average end-to-end delay and throughput of network.

In [16], the researchers increase the performance of AODV routing protocol that validated through ns-2. Mobility characteristics are added to make the AODV protocol suitable to VANETs. Specifically, these characteristics are direction, acceleration, speed and link communication quality between the vehicles. Thus, these mobility features help to select the best route between source to destination.

Rao et al. [17] proposed an AODV routing protocol with backup routes. In case of communication link failure, their AODV can provide the source mobile node with more than one backup paths to destination node. This offers better throughput rate, reduced the end-to-end delay and increased PDR.

However, these routing protocol often encounter a fundamental problem with their data sources. Specifically, GPS, e-maps and sensors on board are utilised for localisation, which may suffer from insufficient accuracy due to high error rates or failure of GPS. This routing protocol that is primarily based on topology data has limitations for use in driverless cars.

In this paper, we proposed routing protocol of the external communication system of autonomous vehicles which is $\mathrm{V}$ AODV. It has the ability to adapt to a high rate of change in the topology and the density of vehicles.

\section{OVERVIEW OF AD HOC ON DEMAND DISTANCE VECTOR ROUTING}

Ad hoc network can employ a variety routing protocols, AODV is considered one of the dominant routing protocols [13]. AODV is a reactive on demand protocol and does not maintain records of all routes at all time. The route discovery process is initiated when there are packets to be sent from the source vehicle to the destination vehicle. The effect of this is that it significantly reduces overhead communication between mobile nodes. Sequence numbers are utilised in order to ensure the freshness of routing paths.

There are three phases in the AODV routing protocol: 1) data/ information transmission, 2) route discovery and 3) route maintenance [18]. The route discovery process is usually started when a source vehicle needs to send packets to a destination vehicle with no routes to the destination in the routing table of the AODV. The source node then sends out a Route Request (RREQ) packet to its neighbouring vehicles. There are three classes of neighbours that receive the RREG packet from source node and they include: 1) it is the destination node; 2) the receiver vehicle has a path to destination vehicle and 3 ) neither of the preceding cases. The receiver node generates a Route Reply (RREP) packet 
in the first two situations and then sends it back to the preceding vehicle, which will then forward the RREP packet. Once completed, the RREP packet will finally move to the source node across the selected route in which the RREQ packet traverses before the source vehicle will finally obtain a route to the destination vehicle.

In the third neighbour class, the receiver vehicle transmits the RREQ packet to all its neighbours until the destination vehicle obtains the RREQ packet or a node obtains a path to the destination mobile node and the process repeats again. The data transmission phase starts after finishing the route discovery phase. In this phase, the source node will send the packets to the destination node through the selected route/ path.

Conversely, the high mobility of vehicles makes it possible for some vehicles to leave the radio coverage area range and the communication links break, which usually leads in the expiration of the transmission. When this situation occurs, the route maintenance phase in the AODV will quickly initiate a call for a repair / find to be executed on the broken route or to obtain a new path to the destination vehicle. However, initially the vehicle that has a broken path generates a Route Error (RERR) packet and return this to the source vehicle. When the RERR packet has been received, the source node will now need to examine its routing table to identify whether there is a previous route to the destination vehicle in that zone. If it discovers that a previous route to the destination node exists, the source node will then choose this route for data transmission. Alternatively, the source vehicle needs to rediscover a path to the destination vehicle and continues with the transmission of the packets.

The RREQ packet is moved from the source node to its neighbours in the route discovery phase and then move again from the neighbours to their new neighbours. The RREQ packet is finally flooded to large portion of the wireless network; this occurrence normally leads to a heavy routing overhead with accompanying large bandwidth consumption between vehicles. Moreover, the source node has the ability to discover more than a single route to the destination node and will then select the route which has the lowest sequence number or the route with the fewest number of hops. Besides, the route may not even last sufficiently long in order to complete the transmission of control data and sensitive information. This is the consequence of highly dynamic topologies in the external communication of autonomous vehicles.

As indicated above, the direct application of MANET routing protocols to VANETs may thus result in poor communication performance. However, AODV usually has a better communication performance compared to other routing protocols as a result of its ability to respond to changes in network quickly and also because of its features of creating paths on demand. To obtain a better performance on AODV in Vehicle Communication (VC), there has been some recent work.

Nomoodiri et al. [19] utilised information such as speed and location in order to approximate the link communication lifetime of paths. Their approach has the ability to establish/select the longest lifetime path to delivery packets at destination vehicle. With this strategy, discovered paths become more stable/ reliable at the cost of a high control overhead rate. Li et al. [20] suggested a scheme to modify packet header in the RREQ packet. A packet header is added to RREQ packet between source to destination.
The simulation results show that transmission delay is low, but the PDR is not optimal.

The neighbours are divided into two groups that based on distances forward RREQ messages from nodes to the outside neighbours which possess a high priority comparing with others [21]. In our research, two steps are made to improve AODV routing protocol which are route discovery and route selection in order to improve route stability and decrease overhead rate.

\section{Methodology of ImProved Ad HOC ON DEMAND DisTANCE VECTOR ROUTING}

Our purpose in the improved AODV routing protocol is to make route more stable and reduce overhead and more applicable with VC. To achieve this protocol, two optimisation steps are implemented on the AODV routing protocol; the mobility parameters of vehicles can be obtained from the Global Position System (GPS), e-maps and other on-board sensors which provide direction, position and speed information. These parameters can play a significant role on the state stability of the route between source to destination.

Large speed differences of two vehicles may lead in short link communication time being available for both vehicles and this type of link is easy to break. However, vehicles have the ability to exchange CAMs with one other vehicles within the radio communication range. Hence, the vehicles which are in motion at a relatively similar velocity will remain in radio coverage area for a longer time and thus there will be increased stability in their link, especially when their movement directions overlap.

However, vehicles travelling in opposite directions will move out of the communication range quickly, while vehicles going in the same direction will remain in communication range for a longer time. Thus, the direction of the movement plays an important role in route stability.

Our suggested AODV protocol is demonstrated in detail in the following; the overall architecture of the proposed vehicle AODV (V-ADOV) routing protocol is illustrated in the figure 2.

- $\quad 1^{\text {st }}$ Phase (route request): route request (RREQ) packets are transmitted on an available path between source to destination in that zone. V-AODV has the ability to establish routes that are more stable from other paths to the destination. We can determine the communication link weight for each available path that based on equation 1 .

$$
\begin{array}{rl}
\text { Route }_{\text {Weight }}=W_{v} & *\left|V_{i}-V_{\text {neighbour of } i}\right|+W_{D} \\
& *\left|D_{i}-D_{\text {neighbour of } i}\right|
\end{array}
$$

where, the source vehicle represents by vehicle $i, \mathrm{~W}_{-} \mathrm{v}-$ speed weight factor, $V_{-}$i - speed of vehicle i, $V_{-}$(neighbour of i) speed of vehicle i's neighbour vehicle, W_D - direction weight factor, $\mathrm{D}_{-} \mathrm{i}$ - direction of node $\mathrm{i}, \mathrm{D}_{-}$(neighbour of $i$ )direction of node $i$ 's neighbour vehicle.

- $\quad 2^{\text {nd }}$ Phase (Route selection Node): To broadcast packets in the external communication system of autonomous vehicles, V-AODV needs to select the most stable route between source to destination if the sender node has 
numerous of route to transmitter. The total weight of select stable paths will be updated in the routing table and integrated with each route reply (RREP) for each sent packet. The total weight of a communication link is determined via equation 2 .

Total Link Weight

$$
\begin{aligned}
& =\sum_{j=2}^{M}\left(W_{V} *\left|V_{j}-V_{j-1}\right|+W_{D}\right. \\
& \left.*\left|D_{j}-D_{j-1}\right|\right)
\end{aligned}
$$

where, $M$ - numbers of vehicles in the route between source to destination, $j$-number sequence of vehicles in the routing table, $\mathrm{V}_{-}$- speed of vehicle $j, \mathrm{~V}_{\mathrm{i}-1}$ - speed of the vehicle $j$ 's previous vehicle in the path, $\bar{D}_{j}$ - direction of vehicle $\left.j, \mathrm{D}_{-\mathrm{j}-1}\right)$ - direction of vehicle $j$ precious vehicle in the path.

- $\quad 3^{\text {rd }}$ Phase (Routing maintenance): In this phase, failed communication paths are notified to sender when intermedia nodes report a link failure. In this case, the source node attempts re-transmission of the failed packets and select another stable path that based on available paths in routing table.

\section{SimULATION RESULTS}

The proposed routing protocol V-AODV is tested by utilising various performance metrics such as PDR, delay rate and control overhead rate. To determine the efficiency of the V-ADOV routing protocol performance metrics are measured under specific communication conditions. Moreover, its performance is compared with original AODV to evaluate the efficiency of the proposed $\mathrm{V}$-AODV. In addition, the factors considered during the testing of the V-AODV performance are traffic packets rate, traffic density rate and the speed of autonomous vehicles.

The performance of $\mathrm{V}$-AODV is compared with AODV with network simulator version 2 (ns-2). Mobility and traffic models are required to evaluate the performance of the proposed $\mathrm{V}$ AODV protocol. The performance metrics are determined from the output files of ns-2, mainly a text trace file and network animator (NAM). The AWK language is employed to analyse and determine metrics such as time delay and PDR [22]. The performance of the proposed routing protocol and AODV is compared for a variety of vehicles numbers as shown in table 1 .

\section{Source Vehicle}

\section{Destination Vehicle}

Data Transmission
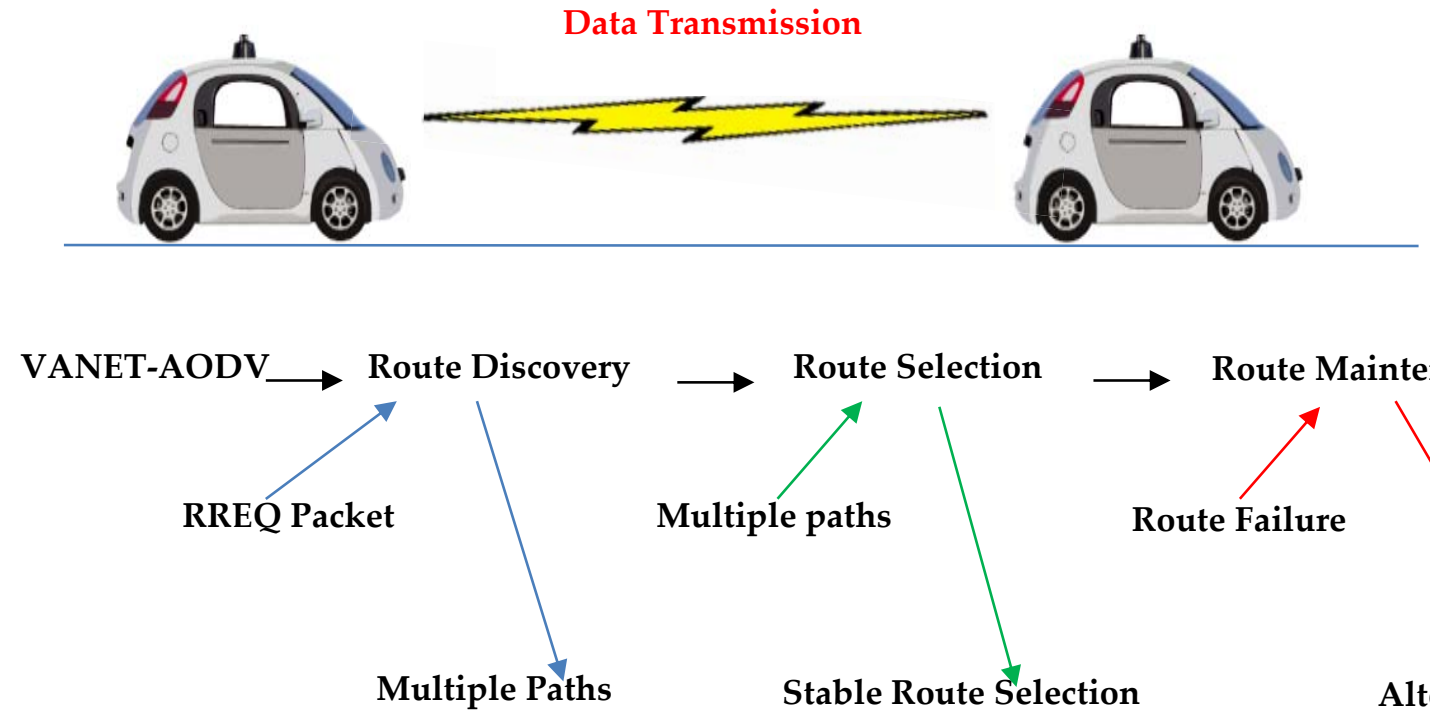

Route Maintenance

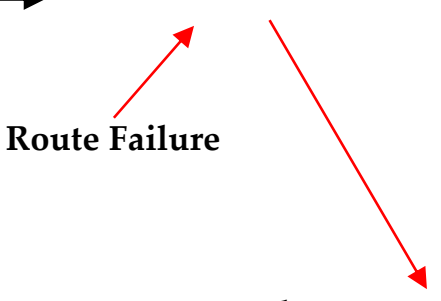

Alternate Route

Fig. 2: Shows Lifecycle of Routing Protocol in VANETs. 
This proposed routing protocol includes both mobility and traffic between mobile nodes in that radio coverage area. It then calculates weight of the all available communication links between the source and the destination. The V-ADOV protocol will select the smallest communication link weight to send packets between two nodes. This it will reduce load on nodes that are not part of these links and allow to reduce the overall control overhead and delay of the external communication system of autonomous vehicles.

The control overhead and time delay of the proposed VAODV routing protocol are calculated and compared with original AODV under various density vehicles in table 1.

Table 1. Performance Metrics with Density of Vehicles

\begin{tabular}{|ccccc|}
\hline \multicolumn{2}{c}{ AODV } & \multicolumn{2}{c|}{ V-AODV } \\
$\begin{array}{c}\text { Vehicle } \\
\text { Number }\end{array}$ & $\begin{array}{c}\text { Control } \\
\text { Overhead }\end{array}$ & Delay & $\begin{array}{c}\text { Control } \\
\text { Overhead }\end{array}$ & Delay \\
\hline 50 & 12939 & $0.188 \mathrm{~s}$ & 3832 & $0.186 \mathrm{~s}$ \\
\hline 100 & 13021 & $0.357 \mathrm{~s}$ & 3798 & $0.295 \mathrm{~s}$ \\
\hline 150 & 14304 & $0.316 \mathrm{~s}$ & 7216 & $0.304 \mathrm{~s}$ \\
\hline 200 & 18137 & $0.397 \mathrm{~s}$ & 11427 & $0.356 \mathrm{~s}$ \\
\hline
\end{tabular}

According to the results in table 1 , we can easily notice that $\mathrm{V}$ AODV can reduce burden rate on the external communication system of autonomous vehicles. In addition, the proposed routing protocol can reduce burden rate and time delay on vehicle network by up to $55 \%$ and $10 \%$ respectively.

Whereas, table 2 shows the performance of the routing protocol with different vehicles travelling at different speeds. Specifically, PDR and time delay are measured and compared for $\mathrm{V}-\mathrm{AODV}$ and for the original AODV under certain conditions. vehicle speed.

Table 2. Performance Metrics with Speed of Vehicles.

\begin{tabular}{|ccccc|}
\hline & \multicolumn{1}{c}{ Original AODV } & \multicolumn{2}{c|}{ V-AODV } \\
$\begin{array}{c}\text { Vehicle } \\
\text { Speed }\end{array}$ & PDR & Delay & PDR & Delay \\
\hline $15 \mathrm{k} / \mathrm{h}$ & $87.675 \%$ & $0.215 \mathrm{~s}$ & $90.480 \%$ & $0.078 \mathrm{~s}$ \\
\hline $25 \mathrm{k} / \mathrm{h}$ & $92.068 \%$ & $0.439 \mathrm{~s}$ & $92.135 \%$ & $0.405 \mathrm{~s}$ \\
\hline $30 \mathrm{k} / \mathrm{h}$ & $85.025 \%$ & $0.358 \mathrm{~s}$ & $87.471 \%$ & $0.261 \mathrm{~s}$ \\
\hline
\end{tabular}

The delivery rate of sent/transferred packets of $\mathrm{V}$-AODV slightly better than original AODV. In more details, V-AODV can improve rate of packet delivery between vehicles by up to $6 \%$. In addition, the time delay of V-AODV is up to $30 \%$ less than the original protocol. Thus, density and speed of vehicles have direct impact on effectiveness and efficiency of the routing protocol.

\section{CONCLUSION}

In this paper, we have proposed a routing protocol for VANETs called $\mathrm{V}$-AODV. It enhances the original AODV routing protocol to adopt with the external communication system of self-driving and semi-autonomous vehicles.

This improved efficiency routing protocol for VANETs is utilising geolocation data such as GPS. We conclude form the simulation results that the proposed VAODV routing protocol that based on link's information is more efficient than protocol based on topology information.

The experimental simulation results demonstrate that the VAODV is a worthwhile enhancement for the external communication system of autonomous and semi-autonomous vehicles. V-AODV protocol allows to significantly reduce the control overhead and delay, while at the same time improving the PDR on VANETs. Future work should explore the performance of $\mathrm{V}$-AODV in a larger network context, with different vehicle densities and under heterogeneous traffic conditions.

\section{ACKNOWLEDGEMENTS}

This work has been supported by CHIST-ERA under the UserCentric Security, Privacy and Trust in the Internet of Things topic through the SPIRIT project, funded via EPSRC grant $\mathrm{EP} / \mathrm{P} 016006 / 1$.

\section{REFERENCES}

[1] K. Ali Alheeti, A. Gruebler, and K. McDonald-Maier, "Intelligent Intrusion Detection of Grey Hole and Rushing Attacks in Self-Driving Vehicular Networks," Computers, vol. 5, no. 3, p. 16, Jul. 2016.

[2] K. M. A. Alheeti, A. Gruebler, and K. D. McDonald-Maier, "On the detection of grey hole and rushing attacks in self-driving vehicular networks," in 2015 7th Computer Science and Electronic Engineering Conference (CEEC), 2015, pp. 231236.

[3] K. M. A. Alheeti and K. McDonald-Maier, "An intelligent intrusion detection scheme for self-driving vehicles based on magnetometer sensors," in 2016 International Conference for Students on Applied Engineering (ISCAE), 2016, pp. 75-78.

N. Garg and P. Rani, "An improved AODV routing protocol for VANET ( Vehicular Ad-hoc Network )," vol. 4, no. 6, pp. 18851890, 2015.

C. E. Perkins, Ad hoc networking. Addison-Wesley, 2001.

$\mathrm{K}$. Ravi and K. Praveen, "AODV routing in VANET for message authentication using ECDSA," Int. Conf. Commun. Signal Process. ICCSP 2014 - Proc., pp. 1389-1393, 2014.

M. Watfa, K. Klinger, L. Tosheff, K. C. Lee, U. Lee, and M. Gerla, "Advances in Vehicular Ad-Hoc Networks: Developments and Challenges InformatIon scIence reference Survey of Routing Protocols in Vehicular Ad Hoc Networks." D. B. Johnson and D. A. Maltz, "Dynamic Source Routing in Ad Hoc Wireless Networks,” Mob. Comput., pp. 153-181.

[9] S. Jaap, M. Bechler, and L. Wolf, "Evaluation of Routing Protocols for Vehicular Ad Hoc Networks in Typical Road Traffic Scenarios," in Proc of the 11th EUNICE Open European Summer School on Networked Applications, 2005, pp. 584-602.

[10] L. X. SUN Xi, "Application of VANET to City Road Trafic Management," J. Shaanxi Univ. Sci. Technol. Sci. Ed., vol. 26, pp. 107-109, 2008.

[11] C. E. Perkins and E. M. Royer, "Ad-hoc on-demand distance vector routing," Proc. - WMCSA'99 2nd IEEE Work. Mob. Comput. Syst. Appl., pp. 90-100, 1999.

[12] C. E. P. and E. M. Royer, "As-Hoc On Demand Distance Vector Routing," Internet Draft , Internet Eng. task Force, 2001. 
[13] B. Ding, Z. Chen, Y. Wang, and H. Yu, "An improved AODV routing protocol for VANETs," in 2011 International Conference on Wireless Communications and Signal Processing (WCSP), 2011, pp. 1-5.

[14] D. Sutariya and S. Pradhan, "An Improved AODV Routing Protocol for VANETs in City Scenarios," Structure, pp. 575$581,2012$.

[15] N. I. Abbas, M. Ilkan, and E. Ozen, "Fuzzy approach to improving route stability of the AODV routing protocol."

[16] Huaqun Guo, F. B. A. Thani, Wai Choong Wong, and Yongdong Wu, "An optimized routing protocol for vehicular ad hoc networks," in TENCON 2010 - 2010 IEEE Region 10 Conference, 2010, pp. 245-250.

[17] M. Rao and N. Singh, "An Improved Routing Protocol (AODV nthBR) for Efficient Routing in MANETs," Springer, Cham, 2014, pp. 215-223.

[18] O. Abedi, M. Fathy, and J. Taghiloo, "Enhancing AODV Routing Protocol 4sing Mobility Parameters in VANET =," Comput. Syst. Appl. 2008. AICCSA 2008. IEEE/ACS Int. Conf. on. IEEE, pp. 229-235, 2008.

[19] V. Namboodiri, M. Agarwal, and L. Gao, "A Study on the Feasibility of Mobile Gateways for Vehicular Ad-hoc Networks," Proc. 1st ACM Int. Work. Veh. ad hoc networks. ACM, 2004.

[20] B. Li, Y. Liu, and G. Chu, "Optimized AODV routing protocol for Vehicular Ad hoc Networks," in 2010 Global Mobile Congress, 2010, pp. 1-4.

[21] O. Abedi, R. Berangi, and M. A. Azgomi, "Improving Route Stability and Overhead on AODV Routing Protocol and Make it Usable for VANET," in 2009 29th IEEE International Conference on Distributed Computing Systems Workshops, 2009, pp. 464-467.

[22] E. H. T. Issariyakul, Introduction to Network Simulator NS2. Springer, 2011. 